\title{
Dynamic Recrystallization and Recovery Behaviors in Austenite of a Novel Fe-1.93Mn-0.07Ni-1.96Cr-0.35Mo Ultrahigh Strength Steel
}

\author{
Yongli Chen $\mathbb{D}^{1,2}$ Yuhua Li, ${ }^{1}$ Xuejiao Zhou $\mathbb{D}^{1},{ }^{1}$ Yueyue Jiang, ${ }^{1,3}$ and Fei Tan ${ }^{1}$ \\ ${ }^{1}$ School of Metallurgical and Materials Engineering, Chongqing University of Science and Technology, Chongqing 401331, China \\ ${ }^{2}$ Chongqing Shanwaishan Blood Purification Technology Co.,Ltd., Chongqing 401123, China \\ ${ }^{3}$ State Key Laboratory of Rolling and Automation, Northeastern University, Shenyang 110819, China
}

Correspondence should be addressed to Yongli Chen; chenyongli@cqust.edu.cn and Xuejiao Zhou; zxj040203017@126.com

Received 14 August 2020; Revised 30 June 2021; Accepted 8 July 2021; Published 14 July 2021

Academic Editor: Tingyue Gu

Copyright (c) 2021 Yongli Chen et al. This is an open access article distributed under the Creative Commons Attribution License, which permits unrestricted use, distribution, and reproduction in any medium, provided the original work is properly cited.

Due to the complex composition and high proportion of alloys in traditional ultrahigh strength steel, the dilemma caused by ultrahigh strength and low toughness in casting and forging processes requiring subsequent heat treatment can be mitigated with an efficient and economical rolling process. In this work, the effect of deformation parameters on dynamic recrystallization (DRX) and dynamic recovery (DRV) is discussed through stress-strain analysis, the DRV mathematical model is obtained, and then the dynamic recrystallization activation energy, Zener-Hollomon equation, and hot working equation are obtained. The critical strain of DRX detected by the P-J method is $\varepsilon_{c} / \varepsilon_{p}=0.631$, which indicates that dynamic recrystallization of this novel steel is relatively easy to achieve by the rolling process. These models and conclusions have potential to be generalized for the formulation of process specification and process configuration without requiring extensive material testing.

\section{Introduction}

Ultrahigh strength steel (UHSS) can reduce steel consumption and improve the safety coefficient so significantly that it is widely used in various industrial fields. However, traditional UHSS is difficult to be smelted as a result of a high alloying addition and a complex composition [1]. Accordingly, the strength of the UHSS is sometimes challenging to match its toughness [2-4]. Eventually, steels exhibit high strength but low toughness. New economic and efficient technology is urgently needed to develop to produce UHSS.

Physical metallurgical behaviors such as work hardening, dynamic restoration, and dynamic recrystallization compete in development during the austenite hot deformation of UHSS, which determines the rheological stress. Rheological stress can reflect the characteristic of UHSS dynamic recrystallization (DRX) [5]. In rolling process, DRX curve can be obtained by three different mechanisms: discontinuous dynamic recrystallization (DDRX), continuous dynamic recrystallization (CDRX), and geometric dynamic recrystallization (GDRX) $[6,7]$. The stacking fault energy of UHSS attributed to Fe-1.93Mn-0.07Ni-1.96Cr-0.35Mo steel is relatively complex, which can be estimated qualitatively from the deformation of work hardening in the flow stress curves [8]. Much attention has been paid to calculate the deformation of carbon steels and austenitic stainless steel [9-11]. Besides, Haridas et al. [12] and Chen et al. [13] studied hot compression effects of deformation temperature, strain rate, and the strain on the microstructural evolution. Zhu et al. [14] investigated the influence of high-temperature deformation and heat treatment on the microstructure of AF1410 in a high Co-Ni aging secondary hardening UHSS. Chen et al. [15] simulated the recrystallization of forging $30 \mathrm{Cr} 2 \mathrm{Ni} 4 \mathrm{MoV}$ ultra-supercritical rotor steel during hot deformation. But little attention has been paid to the high stacking fault energy of the UHSS flow behaviors, which aimed at the efficient direct hot rolling production process. 
According to the difference of strain hardening behaviors between DRV and DRX, Ryan and McQueen [16, 17] defined $\varepsilon_{c}$ as the critical strain where $\theta$ and $\sigma$ begin to deviate from the linear relationship in the $\theta-\sigma$ curve based on the relationship between strain hardening rate $\theta=$ $(\theta=(\mathrm{d} \sigma / \mathrm{d} \varepsilon))$ and true stress $\sigma$, which is called R-M method. Poliak and Jonas et al. [5, 18-20] defined the critical stress $\sigma_{\mathrm{c}}$ as the stress at the point of $|-\partial \theta / \partial \sigma| \min$ on the $(--\partial \theta / \partial \sigma)-\sigma(\theta$ is the strain hardening rate) and determined $\sigma_{\mathrm{c}}$ and $\varepsilon_{\mathrm{c}}$ directly from the stress-strain curve by the P-J method.

This work focuses on the exploitation of a novel bainitic Fe-1.93Mn-0.07Ni-1.96Cr-0.35Mo UHSS by a compact hot rolling process. A thermodynamic simulation experiment for the hot rolling process of experimental steel was carried out to analyze and establish the constitutive equation and dynamic recrystallization model of austenite subjected to the hot deformation. According to P-J method, the critical strain for dynamic recrystallization of austenite was determined, and then the variation law of critical strain under different deformation conditions was studied. Dynamic recrystallization behaviors and austenite refinement were investigated in detail. The theoretical basis and practical guidance for the rolling process of UHSS are provided, which can be expected to have both fundamental and practical significance. We offer an alternative scheme to develop a more economic and efficient process for the UHSS production.

\section{Experimental Materials and Procedures}

2.1. Experimental Materials. Experimental steel was smelted in a $150 \mathrm{~kg}$ vacuum induction furnace. Harmful elements of testing steel, such as S and P, were strictly controlled. The chemical compositions (wt.\%) of experimental samples were 0.23C, 1.95Si, $1.93 \mathrm{Mn}, 0.007 \mathrm{P}, 0.003 \mathrm{~S}, 0.07 \mathrm{Ni}, 1.96 \mathrm{Cr}$, $0.35 \mathrm{Mo}$, and $\mathrm{Nb}+\mathrm{V}+\mathrm{Ti} \leq 2.0$. It was rolled into $10 \mathrm{~mm}$ thick steel sheet by $\phi 450$ hot rolling mills. The finished sheet was air-cooled. Then the specimens were cut horizontally at a quarter width position of the sheet and etched by 4\% (Vol.\%) nitric acid alcohol for metallography after being ground and polished. LEICA 2500M (Leica, Wetzlar, Germany) was applied to observe the microstructure of the examined steel. Tensile tests were performed in a WAW-1000 (SUST Electric Equipment Co., Ltd., Zhuhai, China) to determine the engineering stress.

2.2. Experimental Procedures. The above hot rolled and aircooled sheet was prepared for $\phi 8 \mathrm{~mm} \times 15 \mathrm{~mm}$ thermal simulation samples, and then the experiments were carried out on MMS-300 thermal simulator (RAL of Northeastern University, Shenyang, China). The schematic illustration of single-pass compression experiment is shown in Figure 1. The sample, whose both ends were stained with graphite to reduce friction and avoid the adhesion to achieving homogeneous compression deformation, was heated to $1200^{\circ} \mathrm{C}$ at a heating rate of $10^{\circ} \mathrm{C} \cdot \mathrm{s}^{-1}$ and kept for $180 \mathrm{~s}$ at $1200^{\circ} \mathrm{C}$ to reach a fully austenitized state for the

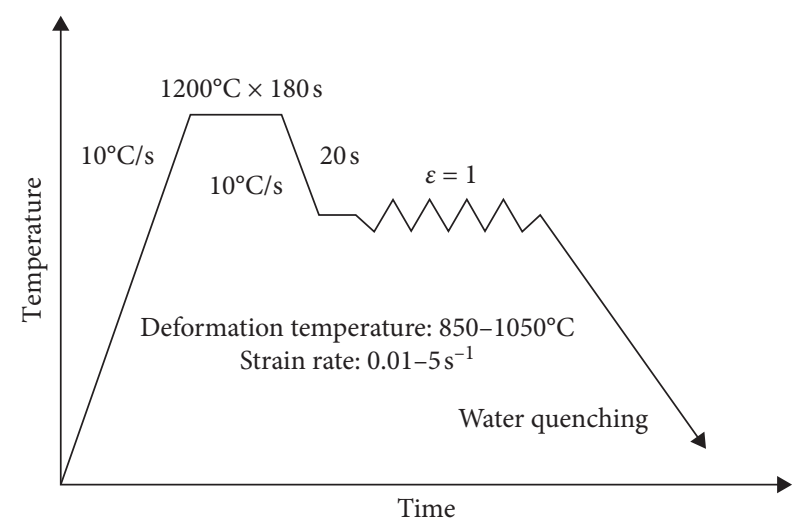

FIgURE 1: Schematic illustration of single-pass compression experiment.

internal structure, and then it was cooled at cooling rate of $10^{\circ} \mathrm{C} \cdot \mathrm{s}^{-1}$ to the different temperature of $850^{\circ} \mathrm{C}, 900^{\circ} \mathrm{C}$, $950^{\circ} \mathrm{C}, 1000^{\circ} \mathrm{C}$, and $1050^{\circ} \mathrm{C}$, respectively. After holding for $20 \mathrm{~s}$ to eliminate the internal temperature gradient, the tested sample was compressed with true strain of 1 at different strain rates of $0.01 \mathrm{~s}^{-1}, 0.1 \mathrm{~s}^{-1}, 0.5 \mathrm{~s}^{-1}, 1 \mathrm{~s}^{-1}$, and $5 \mathrm{~s}^{-1}$, respectively. The hot deformed temperature of $850-1050^{\circ} \mathrm{C}$ is ordinary for direct hot rolling $\left(1250-950^{\circ} \mathrm{C}\right)$ and controlled hot rolling (above $850^{\circ} \mathrm{C}$ ). The strain rate is $0.01-0.5 \mathrm{~s}^{-1}$, which mainly simulates typical hot rolling for the medium sheets rolling (slow rolling rate), hot bar rolling (medium rolling rate), and high speed wire rolling (high rolling rate) in the hot rolling process in the factory. The true strain is 1 ; that is to say, the engineering strain is $63 \%$, which mainly simulates typical high temperature and large reduction deformation in the steel rolling process. Then the effects of deformation parameters such as deformation temperature, deformation rate, and deformation strain on the stress were discussed.

2.3. Microstructural Characterization. Single-pass compressed specimens were cut along the compression axis by wire spark cutting machine. Afterward, they were etched with saturated picric acid alcohol solution with 2 vol.\% Haiou shampoo and 2 wt.\% sodium benzene sulfonate aqueous at room temperature for the characterization of austenite grain boundaries after being ground and polished. Then the morphology of samples was obtained by Leica-DM 2500M (Leica, Wetzlar, Germany) light microscope.

\section{Results and Discussion}

3.1. Original Microstructures and Mechanical Properties. The original microstructure of the examined steel and the results indicated that it is mainly composed of martensite (M) and bainite (B), as shown in Figure 2(a). The mechanical properties of tensile and hardness are presented in Figure 2(b), which shows that yield strength (YS), tensile strength (TS), and elongation $(A)$, are $1260 \mathrm{MPa}, 1570 \mathrm{MPa}$, and $16 \%$, respectively. Thus, the excellent combination of strength and toughness indicates that the rolled UHSS had excellent performance. Therefore, this work offered 


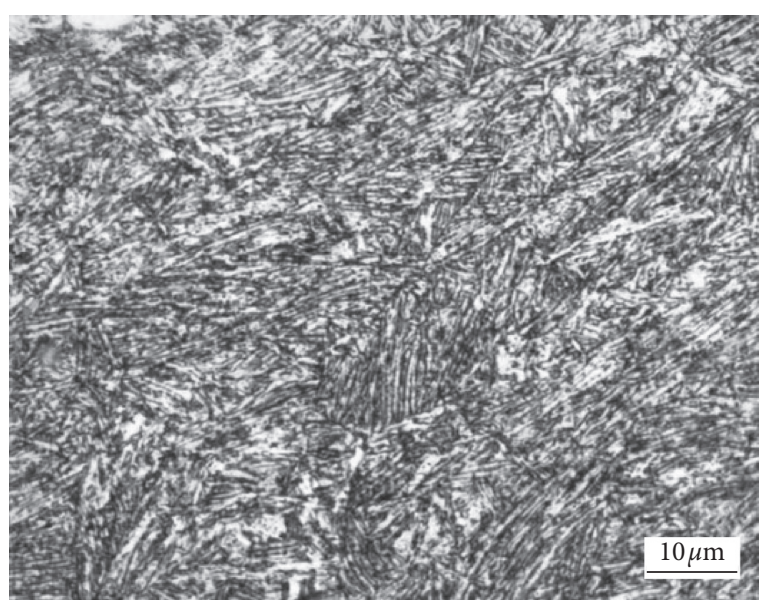

(a)

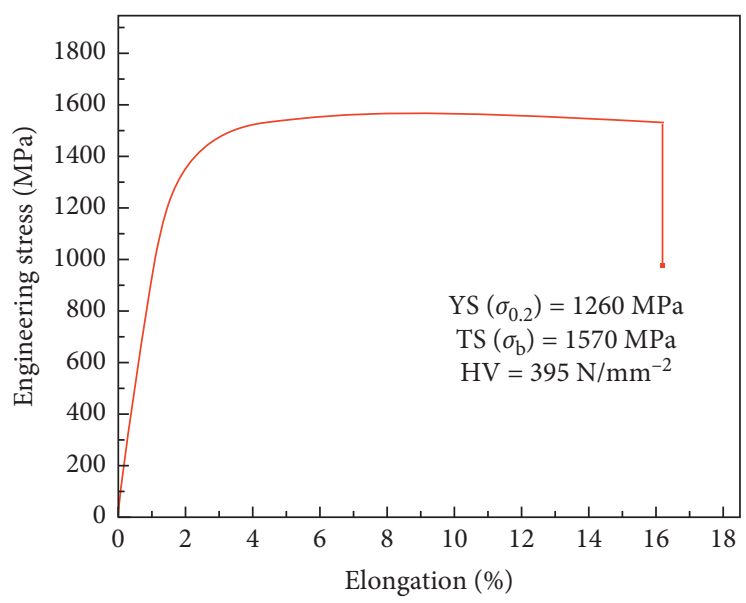

(b)

Figure 2: Original microstructure (a) and mechanical properties (b) of examined steel.

technical and theoretical support for the rolling production of such extraordinary strength-toughness steel.

\subsection{Flow Characteristics and Deformation Mechanisms}

\subsubsection{Effect of Strain Rate on Dynamic Recrystallization.} Figure 3 shows the true stress-true strain curves without friction correction for the experimental steels at $850^{\circ} \mathrm{C}$ and $1050^{\circ} \mathrm{C}$. With a strain rate of $0.01 \mathrm{~s}^{-1}$ at $850^{\circ} \mathrm{C}$, the stressstrain curve is a typical pattern of the steel DRX. When the stress increases to the peak stress, it is meaningful to find that the stress does not increase with the strain, but DRX can still be observed. When the strain rate is increased above $0.1 \mathrm{~s}^{-1}$, the austenite exhibits properties of DRV and work hardening during hot working. It indicates that the strain rate of $0.1 \mathrm{~s}^{-1}$ presents a distinct DRX characteristic at $1050^{\circ} \mathrm{C}$ in the austenite zone.

Comparative analysis of Figures 3(a) and 3(b) shows that DRX of the tested materials is difficult to happen at a relatively high deformation rate. Nevertheless, it is worthy to note that DRX occurs again when the temperature increased to $1050^{\circ} \mathrm{C}$ from $850^{\circ} \mathrm{C}$. This phenomenon indicated that the lower the strain rate is, the lower $\varepsilon_{p}$ and $\varepsilon_{c}$ of the true stresstrue strain curve are, and the DRX is easy to occur at the same deformation temperature. Wang et al. [21] also found that the true stress decreases with the reduced strain rate or the raised temperature. Ansari et al. [22] developed a dislocation-based constitutive model associated with DRV and clarified work hardening mechanisms. As can be seen from Figure 3(b), the true stress-true strain curve can well reflect DRX characteristics at the strain rate of $0.01-0.1 \mathrm{~s}^{-1}$. Further increase of strain rate is conducive to increase $\varepsilon_{p}$ and $\varepsilon_{c}$, while DRX is difficult to occur. When the strain increased to $0.5 \mathrm{~s}^{-1}$, the flow stress reaches $\sigma_{p}$ and then keeps a stable flow state. When the deformation was implemented at a lower temperature and higher strain rate, the increase of $\varepsilon$ could result in the distortion to the lattice of internal grains under the action of external pressure, and DRX did not occur in time for the hardening austenite. At this condition, the stress-strain curve was DRV-shaped.
3.2.2. Effect of Deformation Temperature on Dynamic Recrystallization. Figure 4 displays the true stress-strain curves without friction correction at deformation rates of $0.01 \mathrm{~s}^{-1}$ and $5 \mathrm{~s}^{-1}$. Under the same strain deformation and the strain rate of $0.01 \mathrm{~s}^{-1}$ in Figure 4(a), it is easy to find that the increase of temperature creates slip condition for plastic deformations, and it is also beneficial to decrease the internal resistance of deformation accordingly. Consequently, the deformation resistance is a result of multifactor competition, and deformation temperature is one of the most significant factors. Figure 4(b) indicates that DRX was challenging to occur at a high strain rate. Raising the deformation rate and decreasing the deformation temperature are detrimental to the occurrence of DRX. DRX is more likely to occur at a low strain rate. At the same time, the true stress again increases with the further increase of strain when the deformation degree is relatively large, as shown in Figures 3 and 4 . This phenomenon is because as the deformation increases, the new austenite grains' internal dislocations increase significantly, showing an early stage of work hardening characteristics.

Figure 5(a) is the undeformed metallography and Figures 5(b)-5(d) are the experimental steel at a high temperature of $1050^{\circ} \mathrm{C}$ with the strain rate of $0.5 \mathrm{~s}^{-1}, 0.1 \mathrm{~s}^{-1}$, and $0.01 \mathrm{~s}^{-1}$, respectively. The austenite of undeformed in Figure 5(a) presents a polygonal morphology. From Figure 5(b), the austenite presents a severely flat morphology, but obvious recrystallization is not observed due to DRV. When the strain rate is $0.1 \mathrm{~s}^{-1}$, the hardened austenite is experienced the continuously recrystallizes, the newly formed austenite nucleates along the original grain boundaries, and it presents a deformation and partial recrystallization. As the strain rate is further reduced to $0.01 \mathrm{~s}^{-1}$, the number of recrystallized grains increases significantly. It indicates that austenite DRX transforms decrease with the increase of strain rate. At the same time, it shows that the grains of newly formed austenite in Figures 5(c) and 5(d) are smaller than the original austenite in Figure 5(a). However, as the deformation increases, the newly formed austenite grains are squashed again, but a new 


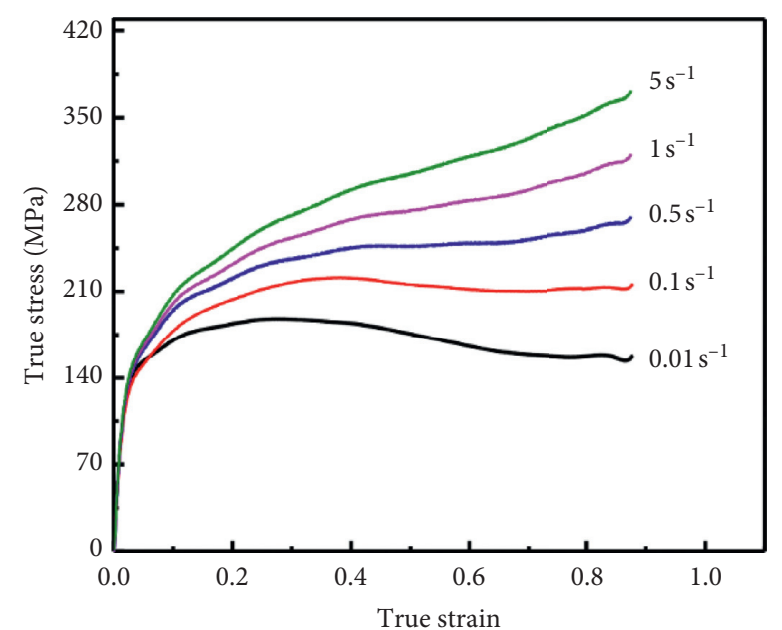

(a)

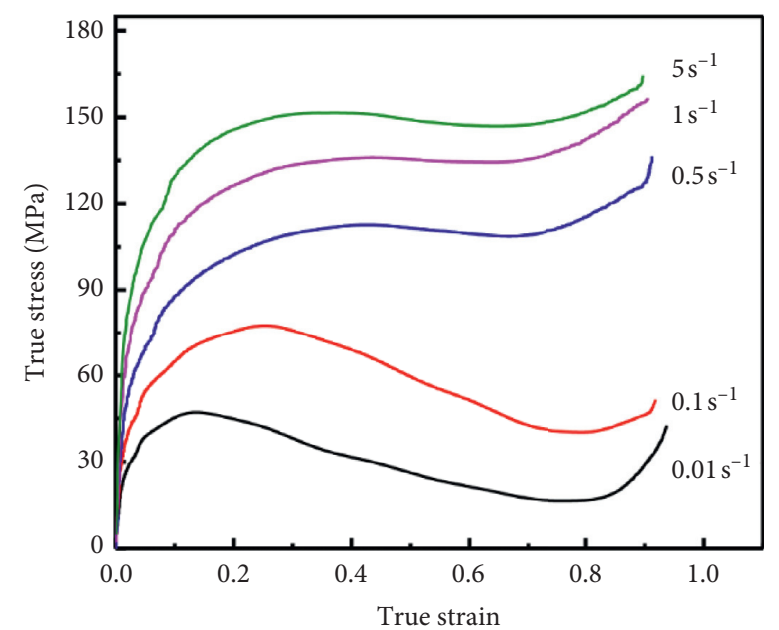

(b)

Figure 3: Influences of strain rates on flow stress at $850^{\circ} \mathrm{C}$ (a) and $1050^{\circ} \mathrm{C} \mathrm{(b)}$.

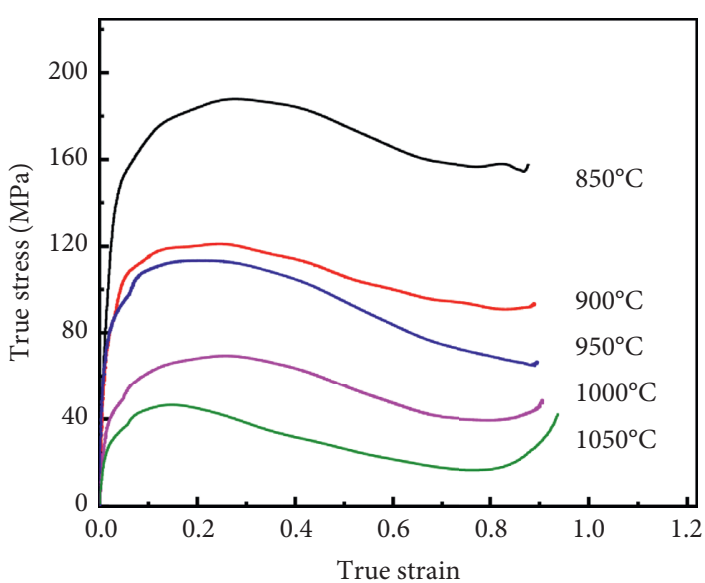

(a)

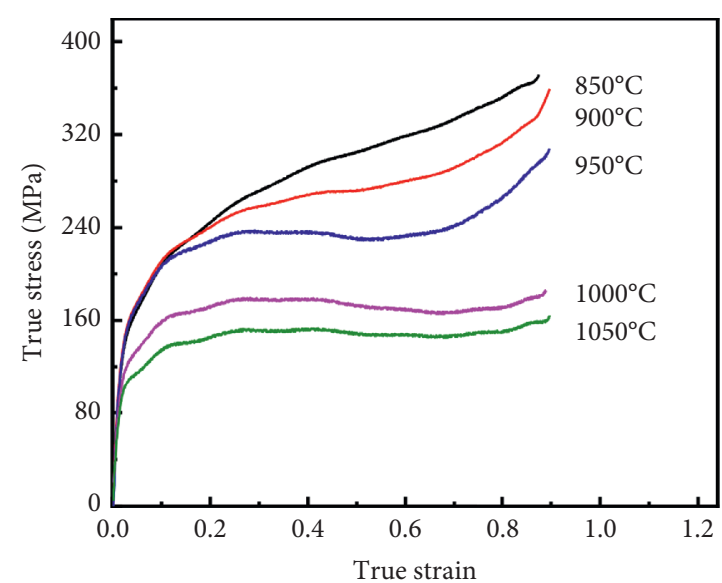

(b)

FIgURE 4: Influences of temperatures on flow stress at strain rates $0.01 \mathrm{~s}^{-1}$ (a) and $0.5 \mathrm{~s}^{-1}$ (b).

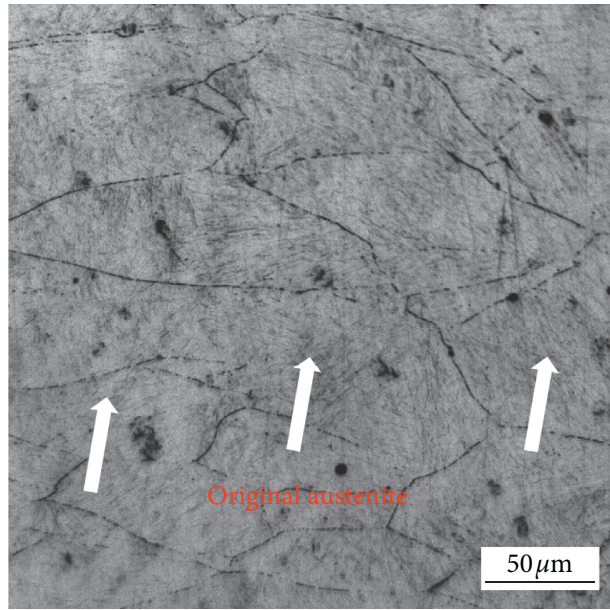

(a)

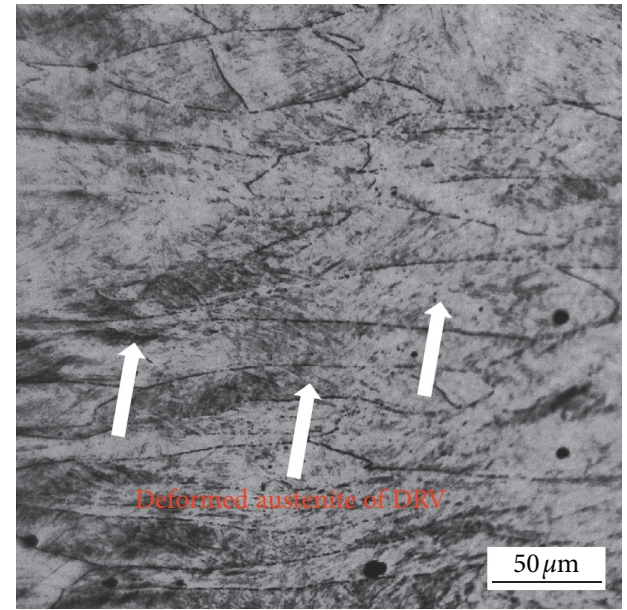

(b)

Figure 5: Continued. 


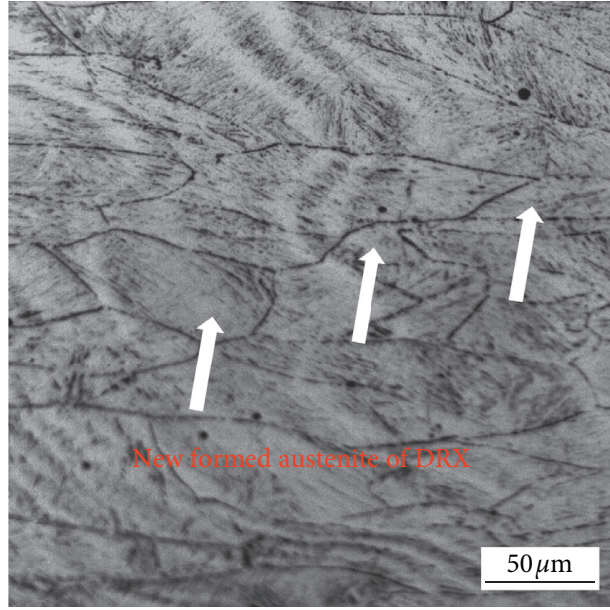

(c)

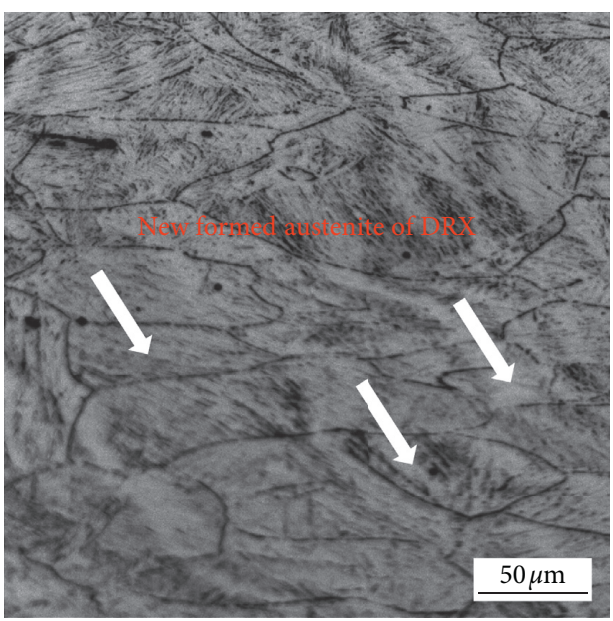

(d)

FIGURE 5: Austenite grain metallography of undeformed (a) strain rate $0.5 \mathrm{~s}^{-1}$ at $1050^{\circ} \mathrm{C}$, (b) strain rate $0.1 \mathrm{~s}^{-1}$ at $1050^{\circ} \mathrm{C}$, and (c) strain rate $0.01 \mathrm{~s}^{-1}$ at $1050^{\circ} \mathrm{C}$.

dynamic recrystallization is not initiated, and a mass of dislocations and subgrains is generated, resulting in the stress increases again with the further increase of strain in Figures 3 and 4.

\subsection{Dynamic Recrystallization Model and Critical Strain.} Under isothermal deformation condition of a higher stress state, the stress-strain curve can be calculated according to the strain rate and deformation temperature by the Zener-Hollomon parameter equation of the temperature compensation strain rate. For the $Z$-parameter model equation of dynamic recrystallization, different scholars proposed different parameter corrections and derivation methods [23-25] and obtained a variety of expressions for the model equations. Through systematic analysis and research, the dynamic recrystallization model can be described as

$$
Z=\dot{\varepsilon} \exp \left(\frac{Q_{d}}{R T}\right)=A\left[\sinh \left(\alpha \sigma_{p}\right)\right]^{n},
$$

where $\dot{\varepsilon}$ is the strain rate, $\mathrm{s}^{-1} ; Q_{d}$ is the activation energy of $\mathrm{DRX}, \mathrm{J} \cdot \mathrm{mol}^{-1}$; $\mathrm{T}$ is the absolute temperature of deformation, $\mathrm{K} ; R$ is the gas constant, $\mathrm{J} \cdot \mathrm{mol}^{-1} \cdot \mathrm{K}^{-1} ; A$ is the structural factor of the model, $\mathrm{s}^{-1} ; \alpha$ is material stress level parameters, $\mathrm{MPa}^{-1} ; \sigma_{p}$ is the peak stress of the true stress-true strain curve, $\mathrm{MPa}$; and $n$ is the stress exponent of the model.

Because the flow stress is dynamic recovery type at lower temperature and higher strain rate, it is appropriate and accurate to analyze the DRX at high temperature by using the experimental data of 950,1000 , and $1050^{\circ} \mathrm{C}$.

3.3.1. Dynamic Recrystallization Model. The Z-parameter determines the stress-strain curve. Sellars et al. [26] proposed a universally applicable hyperbolic sine relation between $\dot{\varepsilon}, T$, and $\sigma$ under isothermal deformation as follows:

$$
\begin{aligned}
A\left[\sinh \left(\alpha \sigma_{p}\right)\right]^{n} & =\dot{\varepsilon} \exp \left(\frac{Q_{d}}{R T}\right)=Z, \\
\dot{\varepsilon} & =A\left[\sinh \left(\alpha \sigma_{p}\right)\right]^{n} \exp \left(-\frac{Q_{d}}{R T}\right) .
\end{aligned}
$$

Kai and Koop [27] suggested that (3) could be simplified to the following model:

$$
\dot{\varepsilon}=A_{2} \sigma_{p}^{n^{\prime}} \exp \left(-\frac{Q_{d}}{R T}\right)
$$

Equation (4) can be simplified by taking the natural logarithm:

$$
\ln \sigma_{p}=\frac{1}{n^{\prime}} \ln \dot{\varepsilon}-\frac{1}{n^{\prime}} \ln A_{2}+\left(\frac{Q_{d}}{n^{\prime} R T}\right) .
$$

According to (5), there is a linear relationship between $\ln$ $\sigma_{p} v s . \ln \dot{\varepsilon}$ and $\ln \sigma_{p} v s .1 / T$, as shown in Figure 6. It can be deduced that $n^{\prime}=\left[\partial \ln \dot{\varepsilon} / \ln \sigma_{p}\right]_{T}, \quad b=\partial \ln \left[\sinh \left(\alpha \sigma_{p}\right)\right] /$ $\partial(1 / T), Q_{d}=n^{\prime} R b$. The values of $n^{\prime}$ in Figure 6(a) and $b$ in Figure 6(b) obtained by regression are shown in Tables 1 and 2. The average value of $n^{\prime}$ is 6.9798 , and the average value of $b$ is 8689.6991 .

According to the values of $n^{\prime}$ and $b$, the activation energy of DRX can be calculated as follows: $Q_{d}=8.314 \times 6.9798 \times$ $8689.6991 \mathrm{~J} \cdot \mathrm{mol}^{-1} \approx 504264 \mathrm{~J} \cdot \mathrm{mol}^{-1}$.

By substituting the DRX activation energy $Q_{d}$ into (1), the $Z$-parameter model is obtained as follows:

$$
Z=\dot{\varepsilon} \exp \left(\frac{504264}{R T}\right)
$$

The value of $A_{2}$ can be determined by (6) and (1). At different strain rates and temperatures, the average value of $A_{2}$ is $0.6089 \mathrm{~s}^{-1}$. Substituting the calculated $A_{2}$ into (1), 


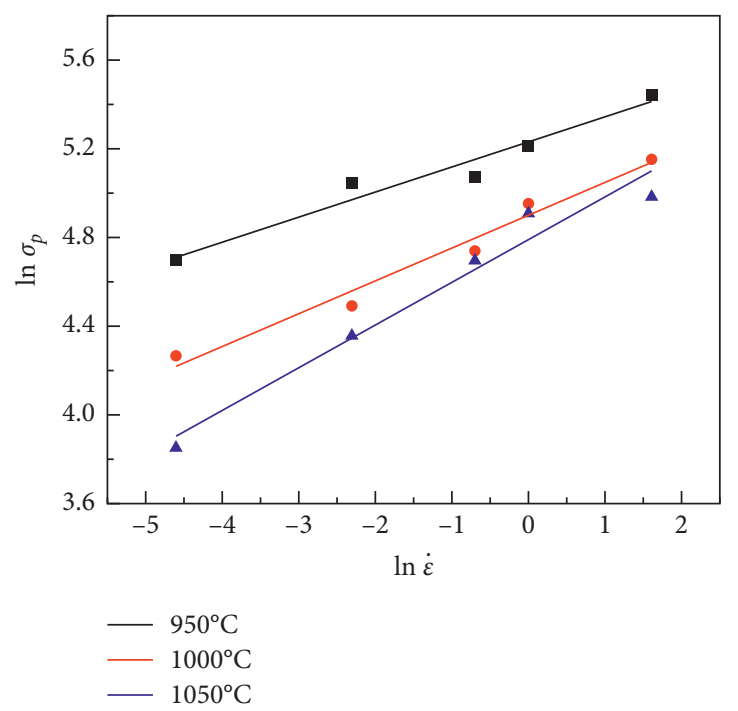

(a)

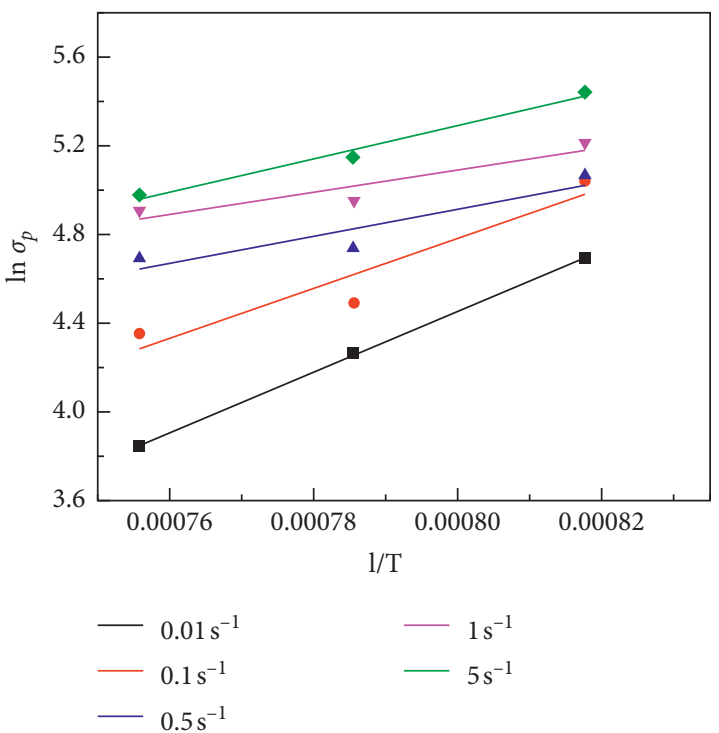

(b)

Figure 6: Plots of $\ln \sigma_{p}$ vs. $\ln \dot{\varepsilon}$ (a) and $\ln \sigma_{p}$ vs. $1 / T$ (b).

TABLE 1: The value of $n^{\prime}$ fitted at different strain rates and temperatures.

\begin{tabular}{llll}
\hline Fitted parameter & $950^{\circ} \mathrm{C}$ & $1000^{\circ} \mathrm{C}$ & $1050^{\circ} \mathrm{C}$ \\
\hline$n^{\prime}$ & 8.9251 & 6.8128 & 5.2015 \\
$R^{2}$ & 0.9527 & 0.9738 & 0.9621 \\
\hline
\end{tabular}

TABLE 2: The value of $b$ fitted at different strain rates and temperatures.

\begin{tabular}{|c|c|c|c|c|c|}
\hline Fitted parameter & $0.01 \mathrm{~s}^{-1}$ & $0.01 \mathrm{~s}^{-1}$ & $0.01 \mathrm{~s}^{-1}$ & $0.01 \mathrm{~s}^{-1}$ & $0.01 \mathrm{~s}^{-1}$ \\
\hline$b$ & 13675.6446 & 11260.3522 & 6059.0263 & 4974.7329 & 7478.7393 \\
\hline$R^{2}$ & 0.9999 & 0.90622 & 0.8501 & 0.8715 & 0.9824 \\
\hline
\end{tabular}

then the hot working equation can be described as follows:

$$
\dot{\varepsilon}=0.6089 \sigma_{p}^{6.9798} \exp \left(-\frac{504264}{R T}\right) .
$$

The peak flow stress $\sigma_{p}$ is expressed as

$$
\sigma_{p}=\left[\frac{1}{0.6089} \dot{\varepsilon} \exp \left(\frac{504264}{R T}\right)\right]^{-6.9798} .
$$

3.3.2. Critical Strain of Dynamic Recrystallization. The P-J method is more accurate in determining the critical conditions of DRX. A typical $(-\partial \theta / \partial \sigma)-\sigma$ curve for UHSS is shown in Figure 7. The flow stress corresponding to the minimum value on the curve is $\sigma_{\mathrm{c}}$ of the DRX process, and the strain corresponding to $\sigma_{c}$ on the true stress-true strain curve is $\varepsilon_{c}$ of the DRX critical strain.

The ratio $\varepsilon_{c} / \varepsilon_{p}$ reflects the relationship between softening and work hardening in the DRX process. If the softening ability of DRX is significant or the work hardening ability is small, the ratio $\varepsilon_{c} / \varepsilon_{p}$ is larger. On the contrary, $\varepsilon_{c} / \varepsilon_{p}$ is lower if the softening capacity of DRX is small or the work hardening ability is significant. The

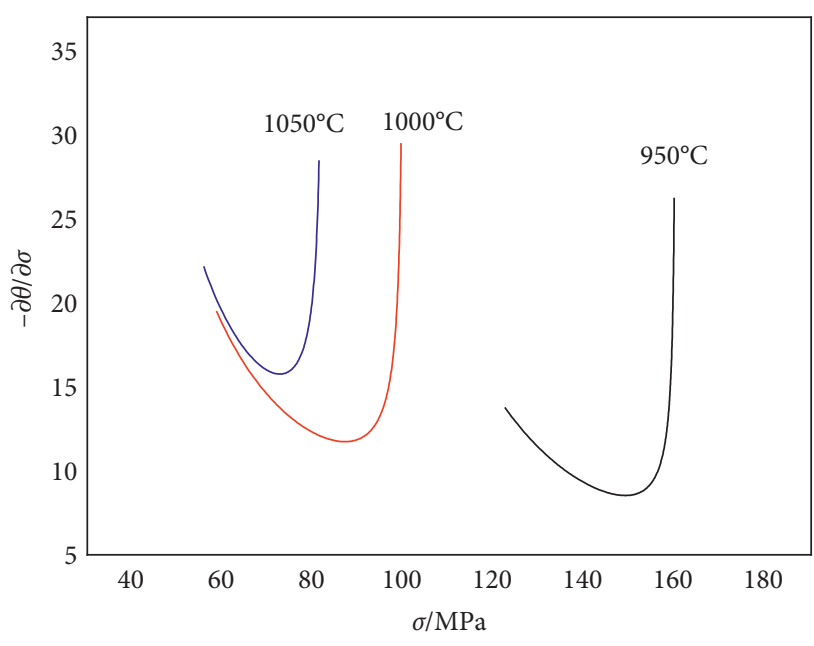

Figure 7: Plots of $(-\partial \theta / \partial \sigma)$ and $\sigma$.

average $\varepsilon_{c} / \varepsilon_{p}$ of DRX is 0.631 by calculation. It is close to the lower limit of empirical formula $\varepsilon_{c} \approx(0.60-0.85) \varepsilon_{p}$, which indicates that DRX is relatively easy to occur in UHSS under the condition of high temperature and low strain rate. 


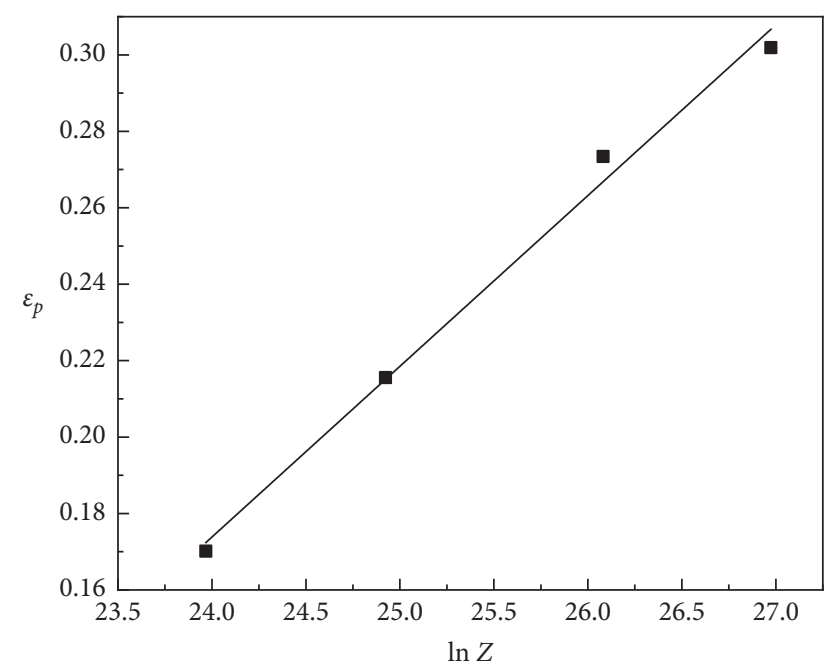

Figure 8: Plot of $\varepsilon_{p}$ versus $\ln Z$.

3.3.3. Relationship between Critical Strain and Z-Parameters. The determination of the critical strain $\varepsilon_{c}$ at the beginning of DRX during hot deformation is of considerable significance to the study of the hot deformation process and the formulation of a reduction schedule. Because $A_{2} \sigma_{p}^{n^{\prime}}$ is equal to $Z$, the relationship between parameter $Z$ and peak strain $\varepsilon_{p}$ and critical strain $\varepsilon_{c}$ can be described as

$$
\begin{gathered}
\varepsilon_{p}=0.045 \ln Z-0.899, \\
\varepsilon_{c}=0.028 \ln Z-0.567 .
\end{gathered}
$$

As can be seen from Figure 8, the empirical formula between $\ln Z$ and $\sigma_{p}$ can be calculated. The relationship between the peak strain $\varepsilon_{p}$ corresponding to the peak stress $\sigma_{p}$ and the dynamic recrystallization critical strain is $\varepsilon_{c} \approx(0.60-0.85) \varepsilon_{p}$. Thus, (10) of the critical strain $\varepsilon_{c}$ and $\ln$ $Z$ of DRX can be further obtained. Consequently, the peak strain $\varepsilon_{p}$ and the critical strain $\varepsilon_{c}$ are linear with $\ln Z$, and the value of parameter $Z$ decreases. Then the peak strain $\varepsilon_{p}$ decreases and the critical deformation $\varepsilon_{c}$ of DRX reduces, which indicates that DRX is easy to occur.

\section{Conclusions}

This paper has studied the influences of deformation parameters on DRX and DRV by stress-strain, and the DRX model has been constructed. The main achievements are summarized as follows:

(1) Dynamic recrystallization and recovery behaviors are obviously affected by deformation temperature and strain rate. The flow stress drops with the reduced strain rate or the raised deformation temperature. Under the corresponding critical strain, above $1050^{\circ} \mathrm{C}$ with a strain rate of $0.01-5 \mathrm{~s}^{-1}$ and $850-1050^{\circ} \mathrm{C}$ with the lower strain rate of $0.01 \mathrm{~s}^{-1}$, DRX can occur in the UHSS, and the dislocations of formed austenite grains increase significantly with increasing the strain.
(2) A DRX model is developed and the calculated activation energy is $504264 \mathrm{~J} \cdot \mathrm{mol}^{-1}$, Zener-Hollomon parameter equation is $Z=\dot{\varepsilon} \exp (504264 / R T)$, and thermal processing equation is $\dot{\varepsilon}=$ $0.6089 \sigma_{p}^{6.9798} \exp (-504264 / R T)$.

(3) The critical strain of DRX detected by the P-J method is $\varepsilon_{c} / \varepsilon_{p}=0.631$, which indicates that DRX of the tested UHSS is relatively easy to occur by hot rolling process. The linear relationship of $\varepsilon_{p}, \varepsilon_{c}$, and $\ln Z$ is as follows: $\varepsilon_{p}=0.45 \mathrm{ln} \quad Z-0.889$ and $\varepsilon_{c}=0.028 \mathrm{ln}$ $Z-0.567$.

\section{Data Availability}

The data used to support the findings of this study are available from the corresponding author upon request.

\section{Conflicts of Interest}

The authors declare that they have no conflicts of interest.

\section{Acknowledgments}

This work was supported by the National Natural Science Foundation of China (nos. 51904052 and 51704055), National Key Research and Development Plan (SQ2020YFF0405436), Science and Technology Research Program of Chongqing Municipal Education Commission (nos. KJQN201801501 and KJQN201901508), Chongqing Special Postdoctoral Science Foundation, Postdoctoral Science Foundation of China (No. 2019M653827XB), Natural Science Foundation of Chongqing, China (nos. cstc2019jcyj-msxmX0106 and cstc2020jcyjmsxm1067), Graduate Science and Technology Innovation Training Program of Chongqing University of Science and Technology (no. YKJCX2020201), and Science and Technology Innovation Training Program for College Students of Chongqing University of Science and Technology (no. 2021197).

\section{References}

[1] Y. Sule, K. Sirin, and E. Kaluc, "Effect of the ion nitriding surface hardening process on fatigue behavior of AISI 4340 steel," Materials Characterization, vol. 59, pp. 351-358, 2007.

[2] H. Matsuda, R. Mizuno, Y. Funakawa, K. Seto, S. Matsuoka, and Y. Tanaka, "Effects of auto-tempering behaviour of martensite on mechanical properties of ultra high strength steel sheets," Journal of Alloys \& Compounds, vol. 577, pp. 661-667, 2013.

[3] Y. Abe, T. Kato, K.-I. Mori, and S. Nishino, "Mechanical clinching of ultra-high strength steel sheets and strength of joints," Journal of Materials Processing Technology, vol. 214, no. 10, pp. 2112-2118, 2014.

[4] C. D. Little and P. M. Machmeier, "High strength fracture resistant weldable steels," US4076525A, 1978.

[5] H. J. McQueen, "Development of dynamic recrystallization theory," Materials Science and Engineering: A, vol. 387-389, pp. 203-208, 2004.

[6] T. Sakai, A. Belyakov, R. Kaibyshev, H. Miura, and J. J. Jonas, "Dynamic and post-dynamic recrystallization under hot, cold 
and severe plastic deformation conditions," Progress in $\mathrm{Ma}$ terials Science, vol. 60, pp. 130-207, 2014.

[7] K. Huang and R. E. Logé, "A review of dynamic recrystallization phenomena in metallic materials," Materials \& Design, vol. 111, pp. 548-574, 2016.

[8] J. Hodowany, G. Ravichandran, A. J. Rosakis, and P. Rosakis, "Partition of plastic work into heat and stored energy in metals," Experimental Mechanics, vol. 40, no. 2, pp. 113-123, 2000.

[9] A. Oudin, P. D. Hodgson, and M. R. Barnett, "EBSD analysis of a Ti-IF steel subjected to hot torsion in the ferritic region," Materials Science and Engineering: A, vol. 486, no. 1-2, pp. 72-79, 2008.

[10] Q. Gao, H. Zhang, H. Li et al., "Hot deformation of aluminaforming austenitic steel: EBSD study and flow behavior," Journal of Materials Science, vol. 54, no. 11, pp. 8760-8777, 2019.

[11] M. Haj, H. Mansouri, R. Vafaei, G. R. Ebrahimi, and A. Kanani, "Hot compression deformation behavior of AISI 321 austenitic stainless steel," International Journal of Minerals, Metallurgy, and Materials, vol. 20, no. 6, pp. 529-534, 2013.

[12] R. S. Haridas, P. Agrawal, S. Thapliyal et al., "Strain rate sensitive microstructural evolution in a TRIP assisted high entropy alloy: experiments, microstructure and modeling," Mechanics of Materials, vol. 156, Article ID 103798, 2021.

[13] X.-M. Chen, Y. C. Lin, D.-X. Wen, J.-L. Zhang, and M. He, "Dynamic recrystallization behavior of a typical nickel-based superalloy during hot deformation," Materials \& Design, vol. 57, pp. 568-577, 2014.

[14] Z. Zhu, W. Yuan, P. Zhang, and R. Zhang, "The influence of high-temperature deformation and heat treatment on microstructure of AF1410 ultra-high strength steel," Steel Research International, vol. 88, no. 12, Article ID 1700135, 2017.

[15] F. Chen, Z. Cui, and S. Chen, "Recrystallization of $30 \mathrm{Cr} 2 \mathrm{Ni} 4 \mathrm{MoV}$ ultra-super-critical rotor steel during hot deformation. Part I: dynamic recrystallization," Materials Science and Engineering: A, vol. 528, no. 15, pp. 5073-5080, 2011.

[16] N. D. Ryan and H. J. Mcqueen, "Hot strength and microstructural evolution of 316 stainless steel during simulated multistage deformation by torsion," Journal of Materials Processing Technology, vol. 36, no. 2, pp. 103-123, 1993.

[17] N. D. Ryan, H. J. Mcqueen, and E. Evangelista, "Dynamic recovery and strain hardening in the hot deformation of type 317 stainless steel," Materials Science and Engineering, vol. 81, pp. 259-272, 1986.

[18] E. I. Poliak and J. J. Jonas, “A one-parameter approach to determining the critical conditions for the initiation of dynamic recrystallization," Acta Materialia, vol. 44, no. 1, pp. 127-136, 1996.

[19] H. J. Mcqueen, S. Yue, N. D. Ryan, and E. Fry, "Hot working characteristics of steels in austenitic state," Journal of Materials Processing Technology, vol. 53, no. 1-2, pp. 293-310, 1995.

[20] H. J. Mcqueen and N. D. Ryan, "Constitutive analysis in hot working," Materials Science \& Engineering: A, vol. 322, no. 12, pp. 43-63, 2002.

[21] K. Wang, D. Wen, J. Li, Z. Zheng, and Y. Xiong, "Hot deformation behaviors of low-alloyed ultrahigh strength steel 30CrMnSiNi2A: microstructure evolution and constitutive modeling," Materials Today Communications, vol. 26, Article ID 102009, 2021.

[22] S. S. Ansari, J. Mukhopadhyay, and S. V. S. N. Murty, "Analysis of stress-strain curves to predict dynamic recrystallization during hot deformation of M300 grade maraging steel," Journal of Materials Engineering and Performance, 2021.

[23] C. Zener and J. H. Hollomon, "Effect of strain rate upon plastic flow of steel," Journal of Applied Physics, vol. 15, no. 1, pp. 22-32, 1944.

[24] S. F. Medina and C. A. Hernandez, "General expression of the Zener-Hollomon parameter as a function of the chemical composition of low alloy and microalloyed steels," Acta Materialia, vol. 44, no. 1, pp. 137-148, 1996.

[25] C. M. Sellars and W. J. M. Tegart, "Hot workability," International Materials Reviews, vol. 17, pp. 1-24, 1971.

[26] C. M. Sellars and W. M. Tegart, "Relationship between strength and structure in deformation at elevated temperatures," Memoires Scientifiques Revue Metallurgie, vol. 63, 1966.

[27] K. Kai and R. Kopp, "Model for integrated process and microstructure simulation in hot forming," Steel Research, vol. 63, no. 6, pp. 247-256, 1992. 